\title{
A comparison of radial versus femoral artery access for acute stroke interventions
}

\author{
Omaditya Khanna, MD, Lohit Velagapudi, BS, Somnath Das, BS, Ahmad Sweid, MD, \\ Nikolaos Mouchtouris, MD, Fadi AI Saiegh, MD, Michael B. Avery, MD, MSc, Nohra Chalouhi, MD, \\ Richard F. Schmidt, MD, Kalyan Sajja, MD, M. Reid Gooch, MD, Stavropoula Tjoumakaris, MD, \\ Robert H. Rosenwasser, MD, and Pascal M. Jabbour, MD
}

\author{
Department of Neurological Surgery, Thomas Jefferson University Hospital, Philadelphia, Pennsylvania
}

\begin{abstract}
OBJECTIVE In this study, the authors aimed to investigate procedural and clinical outcomes between radial and femoral artery access in patients undergoing thrombectomy for acute stroke.

METHODS The authors conducted a single-institution retrospective analysis of 104 patients who underwent mechanical thrombectomy, 52 via transradial access and 52 via traditional transfemoral access. They analyzed various procedural and clinical metrics between the two patient cohorts.
\end{abstract}

RESULTS There was no difference between patient demographics or presenting symptoms of stroke severity between patients treated via transradial or transfemoral access. The mean procedural time was similar between the two treatment cohorts: $60.35 \pm 36.81$ minutes for the transradial group versus $65.50 \pm 29.92$ minutes for the transfemoral group $(p=$ $0.451)$. The mean total fluoroscopy time for the procedure was similar between the two patient cohorts $(20.31 \pm 11.68$ for radial vs $18.49 \pm 11.78$ minutes for femoral, $p=0.898$ ). The majority of patients underwent thrombolysis in cerebral infarction score $2 \mathrm{~b} / 3$ revascularization, regardless of access site ( $92.3 \%$ for radial vs $94.2 \%$ for femoral, $p=0.696$ ). There was no significant difference in the incidence of access site or periprocedural complications between the transradial and transfemoral cohorts.

CONCLUSIONS Acute stroke intervention performed via transradial access is feasible and effective, with no significant difference in procedural and clinical outcomes compared with traditional transfemoral access. Larger studies are required to further validate the efficacy and limitations of transradial access for neurointerventional procedures.

https://thejns.org/doi/abs/10.3171/2020.7.JNS201174

KEYWORDS radial artery catheterization; endovascular; stroke; angiogram; vascular disorders

$\mathrm{H}$ ISTORICALLY, endovascular procedures have been performed via femoral artery catheterization, due to the fact that it is relatively easy to achieve access and the large vessel size allows for the use of widerbore catheters. Although femoral artery catheterization is standard of care among neurointerventionalists, it has been shown to be associated with potential complications such as pseudoaneurysm formation, retroperitoneal hematoma, arteriovenous fistula, and artery occlusion. ${ }^{1-3}$ Furthermore, femoral artery access is painful and uncomfortable for patients, who must remain supine for 4 to 6 hours postoperatively before they are allowed to be mobilized.

Radial artery access has started to gain more widespread use among neurointerventionalists, and it has been shown to be a feasible alternative to femoral artery access for performing a wide range of neuroendovascular procedures with favorable patient satisfaction measures. ${ }^{4-7}$ In the field of interventional cardiology, several large, multicenter randomized controlled trials have demonstrated that radial artery catheterization is associated with fewer access site and procedural complications compared with femoral artery access, ${ }^{8,9}$ as well as improved patient satisfaction ${ }^{10}$ and lower overall costs. ${ }^{11}$ As such, practicing endovascular neurosurgeons at our institution have, of late, begun to adopt transradial access for their interventional procedures with increasing frequency, based on clinical factors more amenable to radial artery catheterization such as a difficult aortic arch configuration, tortuous neck vasculature, and severe obesity (Fig. 1).

In this study, we present our institution's case series of 104 consecutive stroke interventions and report outcome 

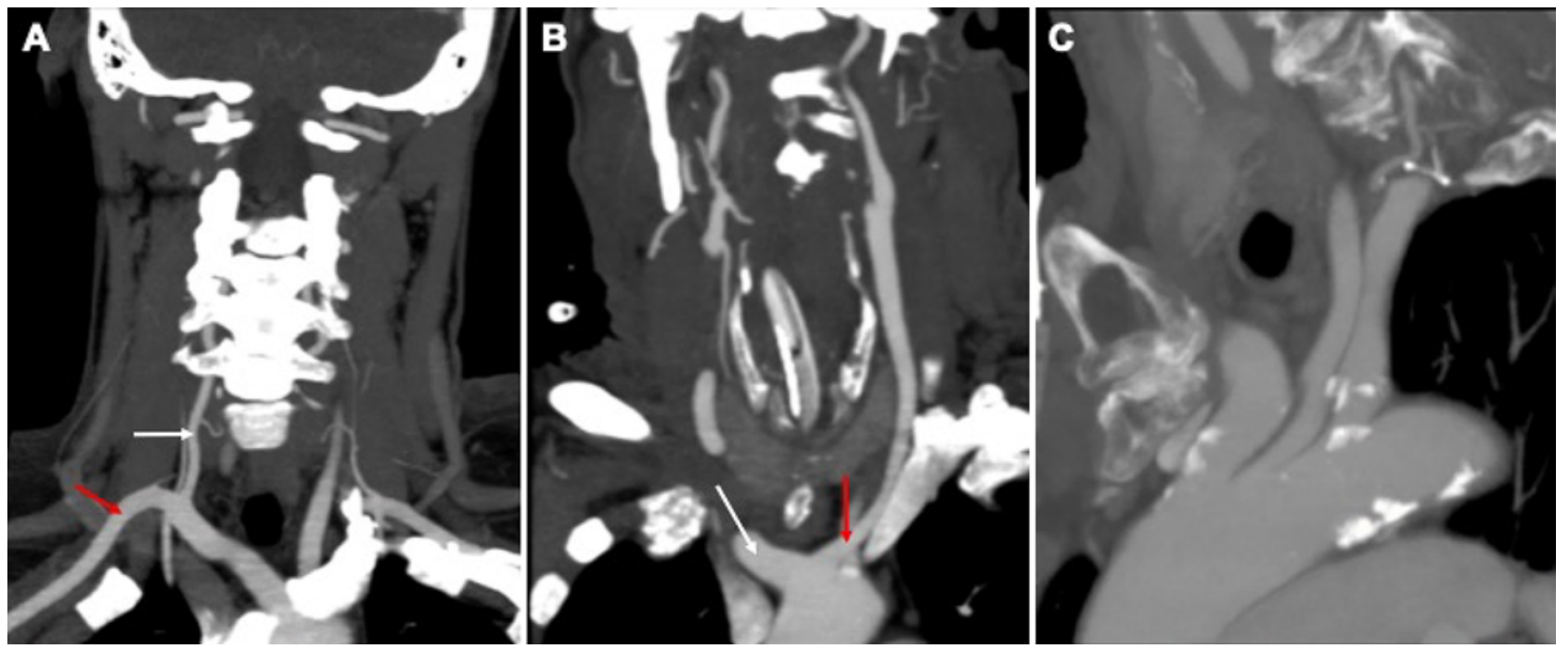

FIG. 1. Illustrative cases of aortic arch configurations more amenable to radial artery catheterization than to femoral access. A: Angiogram obtained in a 38-year-old female with a basilar thrombus, illustrating that patients with vertebrobasilar occlusions can be treated via radial artery access, as it offers a direct access to the vertebral artery (white arrow) via the subclavian artery (red arrow). B: CT angiogram obtained in a 74-year-old patient who presented with a left $\mathrm{M}_{1}$ occlusion. Patients with a bovine aortic arch configuration who require catheterization of the left ICA (red arrow) are excellent candidates for radial artery catheterization, which affords a less tortuous course for access via the subclavian artery (white arrow) compared with femoral artery catheterization. C: Radial artery catheterization is favorable for accessing the right ICA in patients with type 2 or 3 aortic arches, requiring less tension to be built up in the guidewire. Figure is available in color online only.

metrics for the 52 cases that were performed transradial versus 52 that were carried out using traditional femoral artery catheterization during the same time span. Of note, our case series includes the very first procedures performed transradially and illustrates the learning curve associated with adopting a novel treatment paradigm. To our knowledge, this represents the largest case series that compares radial versus femoral artery access for acute stroke intervention.

\section{Methods}

\section{Study Design}

The study protocol was approved by our institutional review board, and the need for informed consent was waived. A retrospective review was conducted of a prospectively maintained single-institution patient database of all acute stroke interventions spanning 1 year from November 2018 (when our first transradial thrombectomy was performed) until December 2019. A total of 104 consecutive cases are included in the overall study cohort, split evenly between 52 consecutive patients who underwent thrombectomy via transradial access and a series of the first 52 consecutive patients who underwent thrombectomy via transfemoral access during the same time frame. Patients who necessitated access site crossover to complete the thrombectomy were excluded from analysis (4 patients required converting from radial to femoral access and 2 patients from femoral to radial access).

All patients presenting with acute stroke undergo CTA at our institution. The CT angiogram is then reviewed by the neuroendovascular fellow and attending surgeon, and a decision is made at that time whether to proceed with thrombectomy via femoral or radial access. Eligible patients are taken for thrombectomy in an interventional suite located adjacent to the CT scanner, which typically commences in short order (less than 15 minutes) after CTA is completed. There were no set inclusion or exclusion criteria for determining whether to pursue femoral versus radial access, and this was left to the discretion of the attending neurosurgeon, although patients who were found to have type 3 aortic arches and tortuous neck vasculature or basilar occlusion, as seen on preprocedural CTA for stroke workup, were more likely to be selected for transradial catheterization. We accessed patients' electronic medical records and collected relevant clinical data pertaining to their clinical presentation, imaging findings on stroke workup, and various relevant procedural metrics.

\section{Radial and Femoral Artery Catheterization Techniques}

All acute stroke interventions included in this study were conducted using conscious sedation. For cases utilizing radial catheterization, the right wrist is prepped and draped, and subcutaneous lidocaine is administered. Radial artery catheterization is achieved using ultrasound guidance via double wall puncture and the Seldinger technique. A 7F slim sheath is introduced, followed by the injection of a radial cocktail consisting of $2000 \mathrm{U}$ of heparin (same dose regardless of whether a patient received intravenous tissue plasminogen activator [tPA]), $5 \mathrm{mg}$ of nicardipine, and $200 \mu \mathrm{g}$ of nitroglycerin to prevent arterial spasm. A radial artery run is performed to evaluate the arterial anatomy, allowing the navigation of a 0.038 -inch Glidewire (Terumo Interventional Systems) into the bra- 
TABLE 1. Patient demographics and clinical presentations of 104 patients who underwent acute stroke intervention via radial or femoral access

\begin{tabular}{lccr}
\hline & $\begin{array}{c}\text { Radial Access } \\
(\mathrm{n}=52)\end{array}$ & $\begin{array}{c}\text { Femoral Access } \\
(\mathrm{n}=52)\end{array}$ & $\mathrm{p}$ Value \\
\hline Sex, M/F & $23 / 29$ & $19 / 33$ & 0.424 \\
\hline Mean age, yrs & $72.9 \pm 13.4$ & $69.5 \pm 16.9$ & 0.200 \\
\hline Mean NIHSS score & $16.9 \pm 7.70$ & $13.12 \pm 7.05$ & 0.922 \\
\hline Vessel occlusion & & & \\
\hline Rt sided & $48.1 \%$ & $46.2 \%$ & $>0.999$ \\
\hline Lt sided & $51.9 \%$ & $53.8 \%$ & $>0.999$ \\
\hline Basilar & $13.5 \%$ & $1.90 \%$ & 0.027 \\
\hline MCA & $76.9 \%$ & $82.7 \%$ & 0.464 \\
\hline ACA & $1.90 \%$ & $3.80 \%$ & $>0.999$ \\
\hline ICA & $5.80 \%$ & $5.80 \%$ & $>0.999$ \\
\hline IV tPA given & & & 0.230 \\
\hline Yes & $34.6 \%$ & $46.2 \%$ & \\
\hline No & $65.4 \%$ & $53.8 \%$ & \\
\hline$>6$ hrs since onset & & & \\
\hline Yes & $36.5 \%$ & $46.0 \%$ & \\
\hline No & $63.5 \%$ & $54.0 \%$ & \\
\hline
\end{tabular}

$\mathrm{ACA}=$ anterior cerebral artery; IV = intravenous; $\mathrm{MCA}=$ middle cerebral artery.

Patients with posterior circulation occlusions were more likely to undergo thrombectomy via radial artery catheterization. Mean values are presented as the mean \pm SD. Boldface type indicates statistical significance.

chial artery. The sheath is then exchanged for a 6F Cook Shuttle (Cook Medical) 90-cm-long sheath, or a 6F 90-cm Ballast long sheath (Balt USA LLC) over the exchange length wire. A small incision is made to facilitate placement of the larger working sheath, as needed. A 5F Sim select (Penumbra, Inc.) is placed inside the Shuttle and is used to navigate to the aortic arch and select the vessel of interest. The Shuttle is advanced over the Sim catheter, parked in the distal common carotid artery or proximal vertebral artery. The Sim catheter is then removed, and the distal guide catheter of choice is inserted inside the long sheath. Mechanical thrombectomy is then begun in earnest using the operator's technique of choice.

After the procedure is complete, the sheath is removed, and a radial artery compression device (TR Band, Terumo Interventional Systems) is applied. The compression band is inflated with air and is incrementally deflated over the course of an hour.

Transfemoral procedures are performed using an $8 \mathrm{~F}$ sheath and conducted in accordance with the operator's preferences based on anatomy and thrombus location. Vessel compression after the procedure was achieved using manual pressure or using Angio-Seal (Terumo Interventional Systems).

\section{Statistical Analysis}

Data were analyzed using descriptive and bivariate statistical methods. Depending on the variable type, normality was tested using a Shapiro-Wilk analysis, chi-square analysis, Fisher's exact test, independent-samples t-test, or Mann-Whitney U-test, as appropriate; $\mathrm{p}<0.05$ was considered statistically significant. Statistical analysis was carried out with IBM SPSS (version 24.0, IBM Corp.).

\section{Results}

A total of 104 consecutive cases were included in our analysis, which included 52 patients who underwent transfemoral access and 52 patients who underwent transradial access (Table 1). There was no significant difference in age or sex between the two cohorts. The mean National Institutes of Health Stroke Scale (NIHSS) score for patients undergoing thrombectomy via transradial access was 16.9 \pm 7.70 and for those who underwent intervention via femoral access it was $13.12 \pm 7.05(\mathrm{p}=0.922)$. There was no significant difference between the radial and femoral groups for time from symptom onset to time of presentation and administration of intravenous tPA. There was no significant difference in the laterality of intervention between the two groups presenting with anterior circulation vessel occlusions or the location of the thrombus itself. However, patients with a basilar occlusion were more likely to be treated via transradial access ( $\mathrm{p}=0.027)$ (Fig. 2).

The mean procedural time (defined as the time between the first and last fluoroscopic image acquisition) was similar between the two treatment cohorts: $60.4 \pm$ 36.8 minutes for the transradial group versus $65.5 \pm 29.9$ minutes for the transfemoral group $(\mathrm{p}=0.451)$. The need for stent placement was not significant based on which access site was used. The total volume of contrast dose administered during the procedure was similar between the two patient cohorts $(107.30 \pm 50.6 \mathrm{ml}$ for the radial group vs $88.5 \pm 48.8 \mathrm{ml}$ for the femoral group, $\mathrm{p}=0.650$ ). Similar revascularization outcomes were achieved between the two treatment cohorts: the majority of procedures yielded thrombolysis in cerebral infarction (TICI) grade $2 \mathrm{~b} / 3$ revascularization in both the transradial $(92.3 \%)$ and transfemoral $(94.2 \%)$ groups $(p=0.696)$, and there was no difference in the mean number of passes performed to achieve these results. The vast majority of thrombectomies in both treatment cohorts were performed via a stent retriever, although transradial access was associated with an increased incidence of requiring a combination of techniques required for recanalization $(51.9 \%$ vs $5.80 \%$, p < 0.001) (Table 2).

Thrombectomies performed via radial and femoral catheterization had similar rates of access site complications. Five patients $(9.6 \%)$ were found to have large, superficial hematomas at the access site in the femoral group compared with 1 patient in the radial group (1.92\%) $(\mathrm{p}=0.093)$. There was 1 patient $(1.92 \%)$ who was found to have a femoral artery pseudoaneurysm that required obliteration with thrombin injection. We did not observe any patients who experienced symptomatic radial artery occlusion, and no patients who underwent radial artery access required any subsequent treatment for access site complications. The incidence of hemorrhagic conversion after thrombectomy was similarly low in both treatment cohorts. Of note, all patients in both treatment cohorts who were found to have hemorrhagic conversion of stroke 

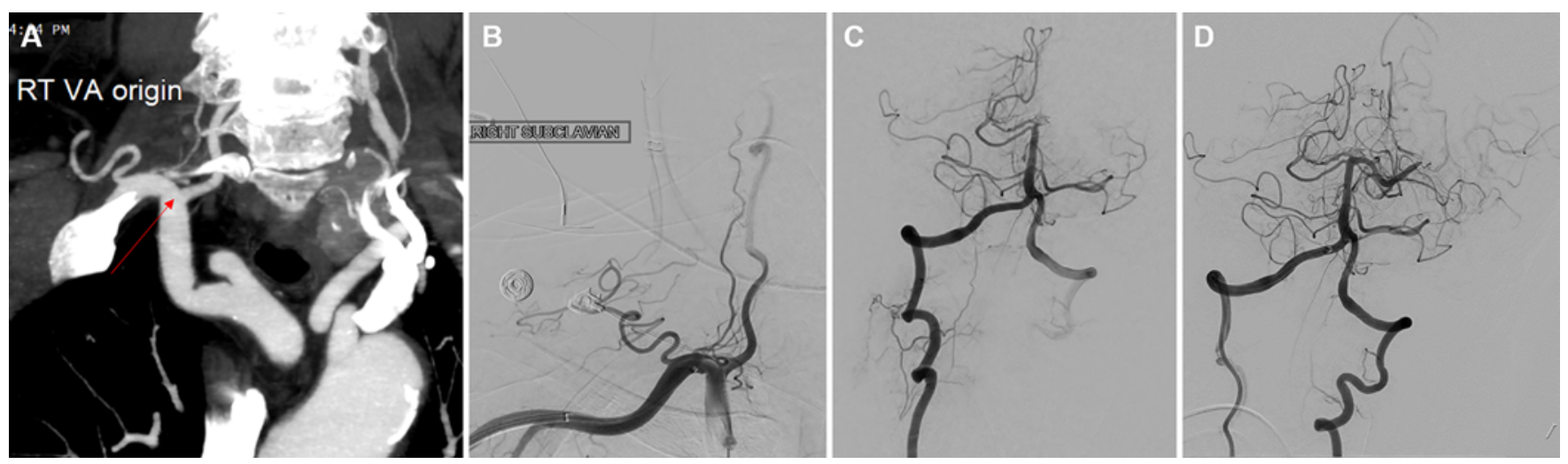

FIG. 2. Illustrative case of a 77-year-old female presenting with progressive lethargy and intractable nausea/vomiting (NIHSS score 21) who was found to have a basilar occlusion. A: CT angiogram showing a type 3 aortic arch and a tortuous innominate and subclavian artery with a sharp-angled origin of the right vertebral artery (RT VA; red arrow). B: A right subclavian injection shows a favorable geometry to catheterize the right vertebral artery. $\mathbf{C}$ and $\mathbf{D}$ : Angiograms obtained prethrombectomy $(\mathrm{C})$ and postthrombectomy (D) that was performed via a stent retriever, resulting in $\mathrm{TICl}$ grade $2 \mathrm{~b}$ revascularization. Figure is available in color online only.

were diagnosed on routine postprocedural imaging, and none required any subsequent treatment. Patients' modified Rankin Scale scores at the time of discharge from the hospital were similar between the radial and femoral cohorts $(3.08 \pm 1.93$ vs $2.96 \pm 1.82, \mathrm{p}=0.831)$.

\section{Discussion}

Currently, radial artery access is not routinely used for neuroendovascular procedures. Despite early case reports detailing the efficacy of the transradial approach, ${ }^{12,13}$ the vast majority of practicing endovascular neurosurgeons still prefer femoral artery access. Our reluctance of using radial artery catheterization for cerebral angiography was primarily due to the perceived difficulty of safely navigating catheters into the cerebral vasculature, which is made even more challenging in patients with anomalous aortic arch anatomy and tortuous vessels. In recent years, there have been many advances in endovascular technology, ${ }^{14}$ with the development of more durable and pliable catheters. Furthermore, several small case series have been

TABLE 2. Summary of procedural and clinical outcomes and complications for patients undergoing acute stroke intervention via radial or femoral access

\begin{tabular}{lccr}
\hline & Radial Access $(\mathrm{n}=52)$ & Femoral Access $(\mathrm{n}=52)$ & $\mathrm{p}$ Value \\
\hline Thrombectomy technique & & & \\
\hline Stent retrieval & $34.6 \%$ & $73.1 \%$ & 0.001 \\
\hline Aspiration & $11.5 \%$ & $21.2 \%$ & 0.185 \\
\hline Stent placement & $1.9 \%$ & $0 \%$ & 0.315 \\
\hline Combination & $51.9 \%$ & $5.80 \%$ & $<0.001$ \\
\hline Mean procedure duration, mins & $60.4 \pm 36.8$ & $65.5 \pm 29.9$ & 0.451 \\
\hline Mean contrast dose, $\mathrm{ml}$ & $107.30 \pm 50.6$ & $88.5 \pm 48.8$ & 0.650 \\
\hline Mean no. of passes & $1.96 \pm 1.21$ & $2.12 \pm 1.30$ & 0.591 \\
\hline TICl revascularization grade & & & \\
\hline 0 & $1.9 \%$ & $1.3 \%$ & 0.315 \\
\hline 1 & $1.9 \%$ & $0 \%$ & 0.315 \\
\hline $2 a$ & $3.8 \%$ & $5.8 \%$ & 0.647 \\
\hline $2 \mathrm{~b} / 3$ & $92.3 \%$ & $94.2 \%$ & 0.696 \\
\hline Access complications & & & \\
\hline Superficial hematoma, $\mathrm{n}(\%)$ & $1(1.9)$ & $5(9.6)$ & 0.093 \\
\hline Pseudoaneurysm, $\mathrm{n}(\%)$ & 0 & $1(1.92)$ & 0.315 \\
\hline Hemorrhagic conversion, $\mathrm{n}(\%)$ & $2(3.85 \%)$ & $3(5.77 \%)$ & 0.647 \\
\hline Mean mRS score at discharge & $3.08 \pm 1.93$ & $2.96 \pm 1.82$ & 0.831 \\
\hline
\end{tabular}

$\mathrm{mRS}=$ modified Rankin Scale.

Mean values are presented as the mean \pm SD. Boldface type indicates statistical significance. 
published that detail the feasibility and utility of using radial access to perform cerebral angiography and mechanical thrombectomy. ${ }^{715}$ This study - to our knowledge, the largest case series to date-highlights the safety and efficacy of radial artery catheterization for acute stroke intervention, with results comparable to those of traditional transfemoral access.

This study includes a patient cohort of 104 stroke interventions that were performed via transradial or transfemoral access. There was no difference in the presenting severity of stroke burden between the two patient cohorts. All studied metrics for procedural outcomes, including procedure time, contrast dose, and TICI revascularization grades, were found to be comparable between the transradial and transfemoral groups. In the treatment of acute stroke, the time from symptom onset to revascularization is paramount in preventing permanent neurological deficits and achieving favorable clinical outcomes. ${ }^{16,17}$ Our case series, which comprises our institution's initial experience with transradial thrombectomy, shows that there is relative equipoise between radial and femoral artery access for duration of needle-to-cerebral reperfusion times. Although we initially began to pursue transradial catheterization only for diagnostic angiography, once we became familiar with this mode of access, we began incorporating it for our acute stroke interventions, and the results of this study illustrate the swift, manageable learning curve associated with adopting radial catheterization into clinical practice, with similar clinical outcomes achieved.

The overall rate of access site complications was low for radial artery access: 1 patient was found to have a superficial hematoma, which resolved with conservative treatment. No patient had persistent symptoms as a result of radial artery catheterization. In the transfemoral group, 5 patients $(9.62 \%)$ were found to have superficial hematomas (albeit none had retroperitoneal hematomas), and 1 patient was found to have femoral artery pseudoaneurysms that required treatment via thrombin injection. Although our case series is small, which, in part, limits achieving statistical significance, the dichotomy in access site complications does serve to highlight the potential for reducing access site complications when radial artery catheterization is utilized over femoral access. Indeed, as the cardiac literature has revealed across multiple, largescale, multicenter trials, radial artery access is associated with an overall significant decrease in access site complications, which has served as the impetus for the field to change its practice such that radial access is preferred over femoral access when feasable. ${ }^{18}$ Larger case series, including, potentially, randomized trials, are needed to assess the incidence of access site complications for radial versus femoral catheterization in patients undergoing neuroendovascular intervention.

Several studies have recently been published that highlight the feasibility and efficacy of using radial artery access for performing a wide range of neurointerventional procedures. ${ }^{4,6,7}$ Chen et al. presented a study of 51 patients, 18 of whom underwent thrombectomy via radial artery access and the remaining 33 via traditional femoral artery access and found that there was no significant difference in operative times, periprocedural complications, or clinical outcomes between the two groups. ${ }^{19}$ Their study also shed light on aortic arch configurations and how radial artery access provided a more favorable trajectory in patients with type 2 or 3 aortic arches or in those who were found to have tortuous vasculature on preoperative CTA. Indeed, our experience with transradial mechanical thrombectomy for anterior circulation occlusions began with using this technique principally for patients with posterior circulation lesions or difficult aortic arch configurations, both of which present favorable geometry for catheterizing desired vessels via radial artery access (Video 1).

VIDEO 1. Various illustrative cases of acute stroke interventions performed via radial artery catheterization. Copyright Omaditya Khanna. Published with permission. Click here to view.

These aortic arch configurations can be evaluated on preprocedural CTA, and we encourage practitioners interested in pursuing transradial thrombectomy to transition their practice using these favorable arch configurations. At our institution, transradial access has now become the default choice for 1) basilar thrombectomy and 2) left-sided internal carotid artery (ICA) thrombectomy with bovine arch configurations. Furthermore, we have been successful in navigating tortuous anatomy via radial artery catheterization using access catheters and guide systems that were initially developed for transfemoral access. As more practitioners adopt the transradial route, we postulate that there will be newer device development that will aid in achieving even improved outcomes.

\section{Conclusions}

In this study, we have shown that acute stroke intervention can be successfully performed via radial artery catheterization, with no difference in clinical outcomes or periprocedural complications compared with traditional transfemoral access. Further studies in a larger patient cohort will need to be performed across several treatment centers to further elucidate equipoise between transradial and transfemoral access for mechanical thrombectomy; specifically, whether radial artery catheterization is associated with a decreased risk of access site complications, as has been convincingly shown in the field of interventional cardiology. We hope that the findings of this study, although limited in scope and case volume, will encourage other neurointerventionalists to pursue radial artery access in their practice, so that our field may advance toward performing a randomized clinical trial to determine whether this is associated with lower morbidities and improved clinical outcomes.

\section{References}

1. Fifi JT, Meyers PM, Lavine SD, et al. Complications of modern diagnostic cerebral angiography in an academic medical center. J Vasc Interv Radiol. 2009;20(4):442-447.

2. Fargen KM, Velat GJ, Lawson MF, et al. Occurrence of angiographic femoral artery complications after vascular closure with Mynx and AngioSeal. J Neurointerv Surg. 2013;5(2): 161-164.

3. Sorenson TJ, Nicholson PJ, Hilditch CA, et al. A lesson from cardiology: the argument for ultrasound-guided femoral artery access in interventional neuroradiology. World Neurosurg. 2019;126:124-128. 
4. Chen SH, Snelling BM, Shah SS, et al. Transradial approach for flow diversion treatment of cerebral aneurysms: a multicenter study. J Neurointerv Surg. 2019;11(8):796-800.

5. Snelling BM, Sur S, Shah SS, et al. Transradial approach for complex anterior and posterior circulation interventions: technical nuances and feasibility of using current devices. Oper Neurosurg (Hagerstown). 2019;17(3):293-302.

6. Khanna O, Sweid A, Mouchtouris N, et al. Radial artery catheterization for neuroendovascular procedures. Stroke. 2019;50(9):2587-2590.

7. Snelling BM, Sur S, Shah SS, et al. Transradial cerebral angiography: techniques and outcomes. J Neurointerv Surg. 2018; 10(9):874-881.

8. Jolly SS, Yusuf S, Cairns J, et al. Radial versus femoral access for coronary angiography and intervention in patients with acute coronary syndromes (RIVAL): a randomised, parallel group, multicentre trial. Lancet. 2011;377(9775): 1409-1420.

9. Andò G, Capodanno D. Radial versus femoral access in invasively managed patients with acute coronary syndrome: a systematic review and meta-analysis. Ann Intern Med. 2015; 163(12):932-940.

10. Hess CN, Krucoff MW, Sheng S, et al. Comparison of quality-of-life measures after radial versus femoral artery access for cardiac catheterization in women: results of the Study of Access Site for Enhancement of Percutaneous Coronary Intervention for Women quality-of-life substudy. Am Heart J. 2015;170(2):371-379.

11. Safley DM, Amin AP, House JA, et al. Comparison of costs between transradial and transfemoral percutaneous coronary intervention: a cohort analysis from the Premier research database. Am Heart J. 2013;165(3):303-309.e2

12. Matsumoto Y, Hongo K, Toriyama T, et al. Transradial approach for diagnostic selective cerebral angiography: results of a consecutive series of 166 cases. AJNR Am J Neuroradiol. 2001;22(4):704-708.

13. Levy EI, Boulos AS, Fessler RD, et al. Transradial cerebral angiography: an alternative route. Neurosurgery. 2002;51(2): 335-342.

14. Rosenwasser RH, Lang M, Tjoumakaris S, Jabbour P. Disruptive innovation in neurovascular disease. Neurosurgery. 2017;64(CN_suppl_1):78-82.

15. McCarthy DJ, Chen SH, Brunet MC, et al. Distal radial artery access in the anatomical snuffbox for neurointerventions: case report. World Neurosurg. 2019;122:355-359.

16. Alawieh A, Vargas J, Fargen KM, et al. Impact of procedure time on outcomes of thrombectomy for stroke. J Am Coll Cardiol. 2019;73(8):879-890.
17. Spiotta AM, Vargas J, Turner R, et al. The golden hour of stroke intervention: effect of thrombectomy procedural time in acute ischemic stroke on outcome. J Neurointerv Surg. 2014;6(7):511-516.

18. Kolkailah AA, Alreshq RS, Muhammed AM, et al. Transradial versus transfemoral approach for diagnostic coronary angiography and percutaneous coronary intervention in people with coronary artery disease. Cochrane Database Syst Rev. 2018;4:CD012318.

19. Chen SH, Snelling BM, Sur S, et al. Transradial versus transfemoral access for anterior circulation mechanical thrombectomy: comparison of technical and clinical outcomes. $J$ Neurointerv Surg. 2019;11(9):874-878.

\section{Disclosures}

The authors report no conflict of interest concerning the materials or methods used in this study or the findings specified in this paper.

\section{Author Contributions}

Conception and design: Jabbour, Khanna, Sweid, Mouchtouris. Acquisition of data: Khanna, Velagapudi, Das, Sweid. Analysis and interpretation of data: Jabbour, Khanna, Velagapudi, Sweid, Mouchtouris, Al Saiegh, Avery, Chalouhi, Schmidt, Sajja, Gooch, Tjoumakaris, Rosenwasser. Drafting the article: Jabbour, Khanna. Critically revising the article: Jabbour, Khanna, Sweid, Mouchtouris, Al Saiegh, Avery, Chalouhi, Schmidt, Sajja, Gooch, Tjoumakaris, Rosenwasser. Reviewed submitted version of manuscript: Khanna, Velagapudi, Das. Approved the final version of the manuscript on behalf of all authors: Jabbour. Statistical analysis: Velagapudi, Mouchtouris. Study supervision: Gooch, Tjoumakaris, Rosenwasser.

\section{Supplemental Information Videos \\ Video 1. https://vimeo.com/443015912.}

\section{Correspondence}

Pascal M. Jabbour: Thomas Jefferson University Hospital, Philadelphia, PA. pascal.jabbour@jefferson.edu. 